\title{
Systems of quadratic diophantine inequalities
}

\author{
par WOLFGANG MÜLLER
}

\begin{abstract}
RÉsumÉ. Soient $Q_{1}, \ldots, Q_{r}$ des formes quadratiques avec des coefficients réels. Nous prouvons que pour chaque $\varepsilon>0$ le système $\left|Q_{1}(x)\right|<\varepsilon, \ldots,\left|Q_{r}(x)\right|<\varepsilon$ des inégalités a une solution entière non-triviale si le système $Q_{1}(x)=0, \ldots, Q_{r}(x)=0$ a une solution réelle non-singulière et toutes les formes $\sum_{i=1}^{r} \alpha_{i} Q_{i}$, $\alpha=\left(\alpha_{1}, \ldots, \alpha_{r}\right) \in \mathbb{R}^{s}, \alpha \neq 0$ sont irrationnelles avec rang $>8 r$.
\end{abstract}

ABSTRACT. Let $Q_{1}, \ldots, Q_{r}$ be quadratic forms with real coefficients. We prove that for any $\epsilon>0$ the system of inequalities $\left|Q_{1}(x)\right|<\epsilon, \ldots,\left|Q_{r}(x)\right|<\epsilon$ has a nonzero integer solution, provided that the system $Q_{1}(x)=0, \ldots, Q_{r}(x)=0$ has a nonsingular real solution and all forms in the real pencil generated by $Q_{1}, \ldots, Q_{r}$ are irrational and have rank $>8 r$.

\section{Introduction}

Let $Q_{1}, \ldots, Q_{r}$ be quadratic forms in $s$ variables with real coefficients. We ask whether the system of quadratic inequalities

$$
\left|Q_{1}(x)\right|<\epsilon, \ldots,\left|Q_{r}(x)\right|<\epsilon
$$

has a nonzero integer solution for every $\epsilon>0$. If some $Q_{i}$ is rational ${ }^{1}$ and $\epsilon$ is small enough then for $x \in \mathbb{Z}^{s}$ the inequality $\left|Q_{i}(x)\right|<\epsilon$ is equivalent to the equation $Q_{i}(x)=0$. Hence if all forms are rational then for sufficiently small $\epsilon$ the system (1.1) reduces to a system of equations. In this case W. Schmidt [10] proved the following result. Recall that the real pencil generated by the forms $Q_{1}, \ldots, Q_{r}$ is defined as the set of all forms

$$
Q_{\alpha}=\sum_{i=1}^{r} \alpha_{i} Q_{i}
$$

where $\alpha=\left(\alpha_{1}, \ldots, \alpha_{r}\right) \in \mathbb{R}^{r}, \alpha \neq 0$. The rational and complex pencil are defined similarly. Suppose that $Q_{1}, \ldots, Q_{r}$ are rational quadratic forms. Then the system $Q_{1}(x)=0, \ldots, Q_{r}(x)=0$ has a nonzero integer solution provided that

\footnotetext{
${ }^{1} \mathrm{~A}$ real quadratic form is called rational if its coefficients are up to a common real factor rational. It is called irrational if it is not rational.
} 
(i) the given forms have a common nonsingular real solution, and either

(iia) each form in the complex pencil has rank $>4 r^{2}+4 r$, or

(iib) each form in the rational pencil has rank $>4 r^{3}+4 r^{2}$.

Recently, R. Dietmann [7] relaxed the conditions (iia) and (iib). He replaced them by the weaker conditions

(iia') each form in the complex pencil has rank $>2 r^{2}+3 r$, or

(iib') each form in the rational pencil has rank $>2 r^{3}$ if $r$ is even and rank $>2 r^{3}+2 r$ if $r$ is odd.

If $r=2$ the existence of a nonsingular real solution of $Q_{1}(x)=0$ and $Q_{2}(x)=0$ follows if one assumes that every form in the real pencil is indefinite (cf. Swinnerton-Dyer [11] and Cook [6]). As noted by W. Schmidt [10] this is false for $r>2$.

We want to consider systems of inequalities (1.1) without hidden equalities. A natural condition is to assume that all forms in the real pencil are irrational. Note that if $Q_{\alpha}$ is rational and $\epsilon$ is small enough, then (1.1) and $x \in \mathbb{Z}^{s}$ imply $Q_{\alpha}(x)=0$. We prove

Theorem 1.1. Let $Q_{1}, \ldots, Q_{r}$ be quadratic forms with real coefficients. Then for every $\epsilon>0$ the system (1.1) has a nonzero integer solution provided that

(i) the system $Q_{1}(x)=0, \ldots, Q_{r}(x)=0$ has a nonsingular real solution,

(ii) each form in the real pencil is irrational and has rank $>8 r$.

In the case $r=1$ much more is known. G.A. MARGulis [9] proved that for an irrational nondegenerate form $Q$ in $s \geq 3$ variables the set $\{Q(x) \mid$ $\left.x \in \mathbb{Z}^{s}\right\}$ is dense in $\mathbb{R}$ (Oppenheim conjecture). In the case $r>1$ all known results assume that the forms $Q_{i}$ are diagonal ${ }^{2}$. For more information on these results see E.D. Freeman [8] and J. Brüdern, R.J. Cook [4].

In 1999 V. BentKus and F. GöTZE [2] gave a completely different proof of the Oppenheim conjecture for $s>8$. We use a multidimensional variant of their method to count weighted solutions of the system (1.1). To do this we introduce for an integer parameter $N \geq 1$ the weighted exponential sum

$$
S_{N}(\alpha)=\sum_{x \in \mathbb{Z}^{s}} w_{N}(x) e\left(Q_{\alpha}(x)\right) \quad\left(\alpha \in \mathbb{R}^{r}\right) .
$$

Here $Q_{\alpha}$ is defined by $(1.2), e(x)=\exp (2 \pi i x)$ as usual, and

$$
w_{N}(x)=\sum_{n_{1}+n_{2}+n_{3}+n_{4}=x} p_{N}\left(n_{1}\right) p_{N}\left(n_{2}\right) p_{N}\left(n_{3}\right) p_{N}\left(n_{4}\right)
$$

\footnotetext{
${ }^{2}$ Note added in proof: Recently, A. GoroDNIK studied systems of nondiagonal forms. In his paper On an Oppenheim-type conjecture for systems of quadratic forms, Israel J. Math. 149 (2004), 125-144, he gives conditions (different from ours) that guarantee the existence of a nonzero integer solution of (1.1). His Conjecture 13 is partially answered by our Theorem 1.1.
} 
denotes the fourfold convolution of $p_{N}$, the density of the discrete uniform probability distribution on $[-N, N]^{s} \cap \mathbb{Z}^{s}$. Since $w_{N}$ is a probability density on $\mathbb{Z}^{s}$ one trivially obtains $\left|S_{N}(\alpha)\right| \leq 1$. The key point in the analysis of Bentkus and GöTzE is an estimate of $S_{N}(\alpha+\epsilon) S_{N}(\alpha-\epsilon)$ in terms of $\epsilon$ alone. Lemma 2.2 gives a generalization of their estimate to the case $r>1$. It is proved via the double large sieve inequality. It shows that for $N^{-2}<|\epsilon|<1$ the exponential sums $S_{N}(\alpha-\epsilon)$ and $S_{N}(\alpha+\epsilon)$ cannot be simultaneously large. This information is almost sufficient to integrate $\left|S_{N}(\alpha)\right|$ within the required precision. As a second ingredient we use for $0<T_{0} \leq 1 \leq T_{1}$ the uniform bound

$$
\lim _{N \rightarrow \infty} \sup _{T_{0} \leq|\alpha| \leq T_{1}}\left|S_{N}(\alpha)\right|=0 .
$$

Note that (1.5) is false if the real pencil contains a rational form. The proof of (1.5) follows closely Bentkus and GöTzE [2] and uses methods from the geometry of numbers.

\section{The double large sieve bound}

The following formulation of the double large sieve inequality is due to Bentkus and Götze [2]. For a vector $T=\left(T_{1}, \ldots, T_{s}\right)$ with positive real coordinates write $T^{-1}=\left(T_{1}^{-1}, \ldots, T_{s}^{-1}\right)$ and set

$$
B(T)=\left\{\left(x_{1}, \ldots, x_{s}\right) \in \mathbb{R}^{s}|| x_{j} \mid \leq T_{j} \text { for } 1 \leq j \leq s\right\} .
$$

Lemma 2.1 (Double large sieve). Let $\mu, \nu$ denote measures on $\mathbb{R}^{s}$ and let $S, T$ be s-dimensional vectors with positive coordinates. Write

$$
J=\int_{B(S)}\left(\int_{B(T)} g(x) h(y) e(\langle x, y\rangle) d \mu(x)\right) d \nu(y),
$$

where $\langle.,$.$\rangle denotes the standard scalar product in \mathbb{R}^{s}$ and $g, h: \mathbb{R}^{s} \rightarrow \mathbb{C}$ are measurable functions. Then

$$
|J|^{2} \ll A\left(2 S^{-1}, g, \mu\right) A\left(2 T^{-1}, h, \nu\right) \prod_{j=1}^{s}\left(1+S_{j} T_{j}\right),
$$

where

$$
A(S, g, \mu)=\int\left(\int_{y \in x+B(S)}|g(y)| d \mu(y)\right)|g(x)| d \mu(x) .
$$


The implicit constant is an absolute one. In particular, if $|g(x)| \leq 1$ and $|h(x)| \leq 1$ and $\mu, \nu$ are probability measures, then

$$
|J|^{2} \ll \sup _{x \in \mathbb{R}^{s}} \mu\left(x+B\left(2 S^{-1}\right)\right) \sup _{x \in \mathbb{R}^{s}} \nu\left(x+B\left(2 T^{-1}\right)\right) \prod_{j=1}^{s}\left(1+S_{j} T_{j}\right) .
$$

Remark. This is Lemma 5.2 in [1]. For discrete measures the lemma is due to E. BombieRI and H. IwANiEc [3]. The general case follows from the discrete one by an approximation argument.

Lemma 2.2. Assume that each form in the real pencil of $Q_{1}, \ldots, Q_{r}$ has rank $\geq p$. Then the exponential sum (1.3) satisfies

$$
S_{N}(\alpha-\epsilon) S_{N}(\alpha+\epsilon) \ll \mu(|\epsilon|)^{p} \quad\left(\alpha, \epsilon \in \mathbb{R}^{r}\right),
$$

where

$$
\mu(t)= \begin{cases}1 & 0 \leq t \leq N^{-2} \\ t^{-1 / 2} N^{-1} & N^{-2} \leq t \leq N^{-1} \\ t^{1 / 2} & N^{-1} \leq t \leq 1 \\ 1 & t \geq 1\end{cases}
$$

Proof. Set $S=S_{N}(\alpha-\epsilon) S_{N}(\alpha+\epsilon)$. We start with

$$
\begin{aligned}
S & =\sum_{x, y \in \mathbb{Z}^{s}} w_{N}(x) w_{N}(y) e\left(Q_{\alpha-\epsilon}(x)+Q_{\alpha+\epsilon}(y)\right) \\
& =\sum_{\substack{m, n \in \mathbb{Z}^{s} \\
m \equiv n(2)}} w_{N}\left(\frac{1}{2}(m-n)\right) w_{N}\left(\frac{1}{2}(m+n)\right) e\left(Q_{\alpha-\epsilon}\left(\frac{1}{2}(m-n)\right)+Q_{\alpha+\epsilon}\left(\frac{1}{2}(m+n)\right)\right) \\
& =\sum_{\substack{m \equiv n(2) \\
|m| \infty,|n| \infty \leq 8 N}} w_{N}\left(\frac{1}{2}(m-n)\right) w_{N}\left(\frac{1}{2}(m+n)\right) e\left(\frac{1}{2} Q_{\alpha}(m)+\frac{1}{2} Q_{\alpha}(n)+\left\langle m, Q_{\epsilon} n\right\rangle\right) .
\end{aligned}
$$

To separate the variables $m$ and $n$ in the weight function write

$$
w_{N}(x)=\int_{B} h(\theta) e(-\langle\theta, x\rangle) d \theta
$$

where $B=(-1 / 2,1 / 2]^{s}$ and $h$ denotes the (finite) Fourier series

$$
h(\theta)=\sum_{k \in \mathbb{Z}^{s}} w_{N}(k) e(\langle\theta, k\rangle) .
$$

Since $w=p_{N} * p_{N} * p_{N} * p_{N}$ we find $h(\theta)=h_{N}(\theta)^{2}$, where

$$
h_{N}(\theta)=\sum_{k \in \mathbb{Z}^{s}} p_{N} * p_{N}(k) e(\langle\theta, k\rangle) .
$$


Now set

$$
\begin{aligned}
a(m) & =e\left(\frac{1}{2}\left(Q_{\alpha}(m)-\left\langle\theta_{1}+\theta_{2}, m\right\rangle\right)\right), \\
b(n) & =e\left(\frac{1}{2}\left(Q_{\alpha}(n)-\left\langle\theta_{1}-\theta_{2}, n\right\rangle\right)\right) .
\end{aligned}
$$

Using (2.5) we find

$$
\begin{aligned}
|S| & =\left|\int_{B} \int_{B} h\left(\theta_{1}\right) h\left(\theta_{2}\right) \sum_{\substack{m \equiv n(2) \\
|m|_{\infty},|n|_{\infty} \leq 8 N}} a(m) b(n) e\left(\left\langle m, Q_{\epsilon} n\right\rangle\right) d \theta_{1} d \theta_{2}\right| \\
& \leq\left(\int_{B}|h(\theta)| d \theta\right)^{2} \sup _{\substack{\theta_{1}, \theta_{2} \in B \\
\theta^{\prime}}}\left|\sum_{\substack{m \equiv n(2) \\
|m| \infty,|n| \infty \leq 8 N}} a(m) b(n) e\left(\left\langle m, Q_{\epsilon} n\right\rangle\right)\right| .
\end{aligned}
$$

Note that $a(m)$ and $b(n)$ are independent of $\epsilon$. Furthermore, by Bessel's inequality

$$
\begin{aligned}
\int_{B}|h(\theta)| d \theta & =\int_{B}\left|h_{N}(\theta)\right|^{2} d \theta \leq \sum_{k \in \mathbb{Z}^{s}}\left(p_{N} * p_{N}(k)\right)^{2} \\
& \leq(2 N+1)^{-s} \sum_{k \in \mathbb{Z}^{s}} p_{N} * p_{N}(k) \leq(2 N+1)^{-s} .
\end{aligned}
$$

Hence

$$
S \ll N^{-2 s} \sum_{\omega \in\{0,1\}^{s}} \sup _{\theta_{1}, \theta_{2} \in B}\left|\sum_{\substack{m \equiv n \equiv \omega(2) \\|m| \infty,|n| \infty \leq 8 N}} a(m) b(n) e\left(\left\langle m, Q_{\epsilon} n\right\rangle\right)\right| .
$$

We are now in the position to apply Lemma 2.1. Denote by $\lambda_{1}, \ldots, \lambda_{s}$ the eigenvalues of $Q_{\epsilon}$ ordered in such a way that $\left|\lambda_{1}\right| \geq \cdots \geq\left|\lambda_{s}\right|$. Then $Q_{\epsilon}=U^{T} \Lambda U$, where $U$ is orthogonal and $\Lambda=\operatorname{diag}\left(\lambda_{1}, \ldots, \lambda_{s}\right)$. Set $\Lambda^{1 / 2}=$ $\operatorname{diag}\left(\left|\lambda_{1}\right|^{1 / 2}, \ldots,\left|\lambda_{s}\right|^{1 / 2}\right), E=\operatorname{diag}\left(\operatorname{sgn}\left(\lambda_{1}\right), \ldots, \operatorname{sgn}\left(\lambda_{s}\right)\right)$ and

$$
\begin{aligned}
& \mathcal{M}=\left\{\left.\Lambda^{1 / 2} U m\left|m \in \mathbb{Z}^{s}, m \equiv \omega(2),\right| m\right|_{\infty} \leq 8 N\right\}, \\
& \mathcal{N}=\left\{\left.E \Lambda^{1 / 2} U m\left|m \in \mathbb{Z}^{s}, m \equiv \omega(2),\right| m\right|_{\infty} \leq 8 N\right\} .
\end{aligned}
$$

Furthermore, let $\mu$ denote the uniform probability distribution on $\mathcal{M}$ and $\nu$ the uniform probability distribution on $\mathcal{N}$. Choose $S_{j}=T_{j}=$ $1+8 \sqrt{s}\left|\lambda_{j}\right|^{1 / 2} N$. Then $x \in \mathcal{M}$ implies $x \in B(T)$ and $y \in \mathcal{N}$ implies $y \in B(S)$. If follows by (2.3) that

$$
\begin{aligned}
&\left|N^{-2 s} \sum_{\substack{m \equiv n \equiv \omega(2) \\
|m| \infty,|n| \infty \leq 8 N}} a(m) b(n) e\left(\left\langle m, Q_{\epsilon} n\right\rangle\right)\right|^{2} \\
& \ll N^{-2 s}\left(\sup _{x \in \mathbb{R}^{s}} A(x)\right)^{2} \prod_{j=1}^{s}\left(1+\left|\lambda_{j}\right| N^{2}\right),
\end{aligned}
$$


where

$$
\begin{aligned}
A(x) & =\#\left\{\left.m \in \mathbb{Z}^{s}|| m\right|_{\infty} \leq 8 N, m \equiv \omega(2), \Lambda^{1 / 2} U m-x \in B\left(2 S^{-1}\right)\right\} \\
& \ll \#\left\{\left.z \in U \mathbb{Z}^{s}|| z\right|_{\infty} \ll N,\left.|| \lambda_{j}\right|^{1 / 2} z_{j}-x_{j} \mid \ll S_{j}^{-1}\right\} \\
& \ll \prod_{j=1}^{s} \min \left(N, 1+\left|\lambda_{j}\right|^{-1} N^{-1}\right) .
\end{aligned}
$$

Hence

$$
S \ll \prod_{j=1}^{s} \tilde{\mu}\left(\left|\lambda_{j}\right|\right)
$$

with $\tilde{\mu}(t)=N^{-1}\left(1+t^{1 / 2} N\right) \min \left(N, 1+t^{-1} N^{-1}\right)$. To prove (2.4) we have to consider the case $N^{-2} \leq|\epsilon| \leq 1$ only. Otherwise the trivial bound $\left|S_{N}(\alpha)\right| \leq 1$ is sufficient. Since $\lambda_{j}=\lambda_{j}(\epsilon)$ varies continuously on $\mathbb{R}^{r} \backslash\{0\}$ and $\lambda_{j}(c \epsilon)=c \lambda_{j}(\epsilon)$ for $c>0$ there exist constants $0<\underline{c}_{j} \leq \bar{c}_{j}<\infty$ such that

$$
\begin{aligned}
\lambda_{j}(\epsilon) & \leq \bar{c}_{j}|\epsilon| & & (1 \leq j \leq s), \\
\underline{c}_{j}|\epsilon| \leq \lambda_{j}(\epsilon) & \leq \bar{c}_{j}|\epsilon| & & (1 \leq j \leq p) .
\end{aligned}
$$

If $N^{-2} \leq|\epsilon| \leq 1$ then $\left|\lambda_{j}\right| \ll 1$ and $\tilde{\mu}\left(\left|\lambda_{j}\right|\right) \ll 1$ for all $j \leq s$. Furthermore, for $j \leq p$ we find $\left|\lambda_{j}\right| \asymp|\epsilon|$ and $\tilde{\mu}\left(\left|\lambda_{j}\right|\right) \ll \max \left(|\epsilon|^{-1 / 2} N^{-1},|\epsilon|^{1 / 2}\right)$. Altogether this yields

$$
S \ll \prod_{j=1}^{p} \tilde{\mu}\left(\left|\lambda_{j}\right|\right) \ll \max \left(|\epsilon|^{-1 / 2} N^{-1},|\epsilon|^{1 / 2}\right)^{p} \ll \mu(|\epsilon|)^{p} .
$$

\section{The uniform bound}

Lemma 3.1 (H. DAVEnPoRT [5]). Let $L_{i}(x)=\lambda_{i 1} x_{1}+\cdots+\lambda_{i s} x_{s}$ be $s$ linear forms with real and symmetric coefficient matrix $\left(\lambda_{i j}\right)_{1 \leq i, j \leq s}$. Denote by $\|$.$\| the distance to the nearest integer. Suppose that P \geq 1$. Then the number of $x \in \mathbb{Z}^{s}$ such that

$$
|x|_{\infty}<P \quad \text { and } \quad\left\|L_{i}(x)\right\|<P^{-1} \quad(1 \leq i \leq s)
$$

$i s \ll\left(M_{1} \ldots M_{s}\right)^{-1}$. Here $M_{1}, \ldots, M_{s}$ denotes the first $s$ of the $2 s$ successive minima of the convex body defined by $F(x, y) \leq 1$, where for $x, y \in \mathbb{R}^{s}$

$$
F(x, y)=\max \left(P\left|L_{1}(x)-y_{1}\right|, \ldots, P\left|L_{s}(x)-y_{s}\right|, P^{-1}\left|x_{1}\right|, \ldots, P^{-1}\left|x_{s}\right|\right) .
$$


Lemma 3.2. Assume that each form in the real pencil of $Q_{1}, \ldots, Q_{r}$ is irrational. Then for any fixed $0<T_{0} \leq T_{1}<\infty$

$$
\lim _{N \rightarrow \infty} \sup _{T_{0} \leq|\alpha| \leq T_{1}}\left|S_{N}(\alpha)\right|=0
$$

Proof. We start with one Weyl step. Using the definition of $w_{N}$ we find

$$
\begin{aligned}
\left|S_{N}(\alpha)\right|^{2} & =\sum_{x, y \in \mathbb{Z}^{s}} w_{N}(x) w_{N}(y) e\left(Q_{\alpha}(y)-Q_{\alpha}(x)\right) \\
& =\sum_{\substack{z \in \mathbb{Z}^{s} \\
|z| \infty \leq 8 N}} \sum_{x \in \mathbb{Z}^{s}} w_{N}(x) w_{N}(x+z) e\left(Q_{\alpha}(z)+2\left\langle z, Q_{\alpha} x\right\rangle\right) \\
& =(2 N+1)^{-8 s} \sum_{m_{i}, n_{i}, z} \sum_{x \in I\left(m_{i}, n_{i}, z\right)} e\left(Q_{\alpha}(z)+2\left\langle z, Q_{\alpha} x\right\rangle\right) .
\end{aligned}
$$

Here the first sum is over all $m_{1}, m_{2}, m_{3}, n_{1}, n_{2}, n_{3}, z \in \mathbb{Z}^{s}$ with $\left|m_{i}\right|_{\infty} \leq N$, $\left|n_{i}\right|_{\infty} \leq N,|z|_{\infty} \leq 8 N$ and $I\left(m_{i}, n_{i}, z\right)$ is the set

$$
\left\{x \in \mathbb{Z}^{s}|| x-n_{1}-n_{2}-\left.n_{3}\right|_{\infty} \leq N,\left|x+z-m_{1}-m_{2}-m_{3}\right|_{\infty} \leq N\right\} .
$$

It is an $s$-dimensional box with sides parallel to the coordinate axes and side length $\ll N$. By Cauchy's inequality it follows that

$$
\begin{aligned}
\left|S_{N}(\alpha)\right|^{4} & \ll N^{-9 s} \sum_{m_{i}, n_{i}, z}\left|\sum_{x \in I\left(m_{i}, n_{i}, z\right)} e\left(2\left\langle x, Q_{\alpha} z\right\rangle\right)\right|^{2} \\
& \ll N^{-3 s} \sum_{|z|_{\infty} \leq 8 N} \prod_{i=1}^{s} \min \left(N,\left\|2\left\langle e_{i}, Q_{\alpha} z\right\rangle\right\|^{-1}\right)^{2} .
\end{aligned}
$$

Here we used the well known bound

$$
\sum_{x \in I_{1} \times \cdots \times I_{s}} e(\langle x, y\rangle) \ll \prod_{i=1}^{s} \min \left(\left|I_{i}\right|,\left\|\left\langle e_{i}, y\right\rangle\right\|^{-1}\right),
$$

where $I_{i}$ are intervals of length $\left|I_{i}\right| \gg 1$ and $e_{i}$ denotes the $i$-th unit vector. Set

$$
\mathcal{N}(\alpha)=\#\left\{\left.z \in \mathbb{Z}^{s}|| z\right|_{\infty} \leq 16 N,\left\|2\left\langle e_{i}, Q_{\alpha} z\right\rangle\right\|<1 / 16 N \text { for } 1 \leq i \leq s\right\} .
$$

We claim that

$$
\left|S_{N}(\alpha)\right|^{4} \ll N^{-s} \mathcal{N}(\alpha)
$$

To see this set

$$
\mathcal{D}_{m}(\alpha)=\#\left\{\left.z \in \mathbb{Z}^{s}|| z\right|_{\infty} \leq 8 N, \frac{m_{i}-1}{16 N} \leq\left\{2\left\langle e_{i}, Q_{\alpha} z\right\rangle\right\}<\frac{m_{i}}{16 N} \text { for } i \leq s\right\},
$$

where $\{x\}$ denotes the fractional part of $x$. Then $\mathcal{D}_{m}(\alpha) \leq \mathcal{N}(\alpha)$ for all $m=\left(m_{1}, \ldots, m_{s}\right)$ with $1 \leq m_{i} \leq 16 N$. Note that if $z_{1}$ and $z_{2}$ are counted 
in $\mathcal{D}_{m}(\alpha)$ then $z_{1}-z_{2}$ is counted in $\mathcal{N}(\alpha)$. It follows that

$$
\begin{aligned}
\left|S_{N}(\alpha)\right|^{4} & \ll N^{-3 s} \sum_{1 \leq m_{i} \leq 16 N} \mathcal{D}_{m}(\alpha) \prod_{i=1}^{s} \min \left(N, \frac{16 N}{m_{i}-1}+\frac{16 N}{16 N-m_{i}}\right)^{2} \\
& \ll N^{-3 s} \mathcal{N}(\alpha) \sum_{1 \leq m_{i} \leq 8 N} \prod_{i=1}^{s} \frac{N^{2}}{m_{i}^{2}} \\
& \ll N^{-s} \mathcal{N}(\alpha) .
\end{aligned}
$$

To estimate $\mathcal{N}(\alpha)$ we use Lemma 3.1 with $P=16 N$ and $L_{i}(x)=2\left\langle e_{i}, Q_{\alpha} x\right\rangle$ for $1 \leq i \leq s$. This yields

$$
\mathcal{N}(\alpha) \ll\left(M_{1, \alpha} \ldots M_{s, \alpha}\right)^{-1},
$$

where $M_{1, \alpha} \leq \cdots \leq M_{s, \alpha}$ are the first $s$ from the $2 s$ successive minima of the convex body defined in Lemma 3.1.

Now suppose that there exists an $\epsilon>0$, a sequence of real numbers $N_{n} \rightarrow \infty$ and $\alpha^{(n)} \in \mathbb{R}^{r}$ with $T_{0} \leq\left|\alpha^{(n)}\right| \leq T_{1}$ such that

$$
\left|S_{N_{n}}\left(\alpha^{(n)}\right)\right| \geq \epsilon .
$$

By (3.1) and (3.2) this implies

$$
\epsilon^{4} N_{n}^{s} \ll\left(\prod_{i=1}^{s} M_{i, \alpha}(n)\right)^{-1}
$$

Since $\left(16 N_{n}\right)^{-1} \leq M_{1, \alpha^{(n)}} \leq M_{i, \alpha^{(n)}}$ we obtain $\epsilon^{4} N_{n}^{s} \ll N_{n}^{s-1} M_{s, \alpha^{(n)}}^{-1}$ and this proves

$$
\left(16 N_{n}\right)^{-1} \leq M_{1, \alpha^{(n)}} \leq \cdots \leq M_{s, \alpha^{(n)}} \ll\left(\epsilon^{4} N_{n}\right)^{-1} .
$$

By the definition of the successive minima there exist $x_{j}^{(n)}, y_{j}^{(n)} \in \mathbb{Z}^{s}$ such that $\left(x_{1}^{(n)}, y_{1}^{(n)}\right), \ldots,\left(x_{s}^{(n)}, y_{s}^{(n)}\right)$ are linearly independent and $M_{j, \alpha^{(n)}}=$ $F\left(x_{j}^{(n)}, y_{j}^{(n)}\right)$. Hence for $1 \leq i, j \leq s$

$$
\begin{aligned}
\left.\mid L_{i}\left(x_{j}^{(n)}\right)-y_{j, i}^{(n)}\right) \mid & \ll N_{n}^{-2}, \\
\left|x_{j, i}^{(n)}\right| & \ll 1 .
\end{aligned}
$$

Since $\left|\alpha^{(n)}\right| \leq T_{1}$ this inequalities imply $\left|y_{j, i}^{(n)}\right| \ll_{T_{1}} 1$. This proves that the integral vectors

$$
W_{n}=\left(x_{1}^{(n)}, y_{1}^{(n)}, \ldots, x_{s}^{(n)}, y_{s}^{(n)}\right) \quad(n \geq 1)
$$

are contained in a bounded box. Thus there exists an infinite sequence $\left(n_{k}^{\prime}\right)_{k \geq 1}$ with $W_{n_{1}^{\prime}}=W_{n_{k}^{\prime}}$ for $k \geq 1$. The compactness of $\left\{\alpha \in \mathbb{R}^{s} \mid T_{0} \leq\right.$ $\left.|\alpha| \leq T_{1}\right\}$ implies that there is a subsequence $\left(n_{k}\right)_{k \geq 1}$ of $\left(n_{k}^{\prime}\right)_{k \geq 1}$ with 
$\lim _{k \rightarrow \infty} \alpha^{\left(n_{k}\right)}=\alpha^{(0)}$ and $T_{0} \leq\left|\alpha^{(0)}\right| \leq T_{1}$. Let $x_{j}=x_{j}^{\left(n_{k}\right)}$ and $y_{j}=y_{j}^{\left(n_{k}\right)}$ for $1 \leq j \leq s$. Then $x_{j}$ and $y_{j}$ are well defined and

$$
y_{j}=\left(L_{1}\left(x_{j}\right), \ldots, L_{s}\left(x_{j}\right)\right)=2 Q_{\alpha^{(0)}} x_{j} \quad(1 \leq j \leq s) .
$$

We claim that $x_{1}, \ldots, x_{s}$ are linearly independent. Indeed, suppose that there are $q_{j}$ such that $\sum_{j=1}^{s} q_{j} x_{j}=0$. Then $\sum_{j=1}^{s} q_{j} y_{j}=0$ by (3.4). This implies $\sum_{j=1}^{s} q_{j}\left(x_{j}, y_{j}\right)=0$ and the linear independence of $\left(x_{j}, y_{j}\right)$ yields $q_{j}=0$ for all $j$. The matrix equation $2 Q_{\alpha^{(0)}}\left(x_{1}, \ldots, x_{s}\right)=\left(y_{1}, \ldots, y_{s}\right)$ implies that $Q_{\alpha^{(0)}}$ is rational. By our assumptions this is only possible if $\alpha^{(0)}=0$, contradicting $\left|\alpha^{(0)}\right| \geq T_{0}>0$. This completes the proof of the Lemma.

Lemma 3.3. Assume that each form in the real pencil of $Q_{1}, \ldots, Q_{r}$ is irrational and has rank $\geq 1$. Then there exists a function $T_{1}(N)$ such that $T_{1}(N)$ tends to infinity as $N$ tends to infinity and for every $\delta>0$

$$
\lim _{N \rightarrow \infty} \sup _{N^{\delta-2} \leq|\alpha| \leq T_{1}(N)}\left|S_{N}(\alpha)\right|=0 .
$$

Proof. We first prove that there exist functions $T_{0}(N) \leq T_{1}(N)$ such that $T_{0}(N) \downarrow 0$ and $T_{1}(N) \uparrow \infty$ for $N \rightarrow \infty$ and

$$
\lim _{N \rightarrow \infty} \sup _{T_{0}(N) \leq|\alpha| \leq T_{1}(N)}\left|S_{N}(\alpha)\right|=0 .
$$

From Lemma 3.2 we know that for each $m \in \mathbb{N}$ there exist an $N_{m}$ with

$$
\left|S_{N}(\alpha)\right| \leq \frac{1}{m} \quad \text { for } \quad N \geq N_{m} \quad \text { and } \quad \frac{1}{m} \leq|\alpha| \leq m .
$$

Without loss of generality we assume that $\left(N_{m}\right)_{m \geq 1}$ is increasing. For $N_{m} \leq N<N_{m+1}$ define $T_{0}(N)=\frac{1}{m}, T_{1}(N)=m$ and for $N<N_{1}$ set $T_{0}(N)=T_{1}(N)=1$. Obviously this choice satisfies (3.5). Replacing $T_{0}(N)$ by $\max \left(T_{0}(N), N^{-1}\right)$ we can assume that $N^{-1} \leq T_{0}(N) \leq 1$. Finally, Lemma 2.2 with $p \geq 1$ yields

$$
\begin{aligned}
\sup _{N^{\delta-2} \leq|\alpha| \leq T_{0}(N)} & \left|S_{N}(\alpha)\right| \\
\ll & \sup _{N^{\delta-2} \leq|\alpha| \leq T_{0}(N)} \mu(|\alpha|)^{p} \ll \max \left(N^{-\delta / 2}, T_{0}(N)^{1 / 2}\right)^{p} \rightarrow 0 .
\end{aligned}
$$

\section{The integration procedure}

In this section we use Lemma 2.2 to integrate $\left|S_{N}(\alpha)\right|$. It is here where we need the assumption $p>8 r$. 
Lemma 4.1. For $0<U \leq T$ set $B(U, T)=\left\{\alpha \in \mathbb{R}^{r}|U \leq| \alpha \mid \leq T\right\}$ and define

$$
\gamma(U, T)=\sup _{\alpha \in B(U, T)}\left|S_{N}(\alpha)\right| .
$$

Furthermore, let $h$ be a measurable function with $0 \leq h(\alpha) \leq(1+|\alpha|)^{-k}$, $k>r$. If each form in the real pencil generated by $Q_{1}, \ldots, Q_{r}$ has rank $\geq p$ with $p>8 r$ and if $\gamma(U, T) \geq 4^{p /(8 r)} N^{-p / 4}$ then

$$
\int_{B(U, T)}\left|S_{N}(\alpha)\right| h(\alpha) d \alpha \ll N^{-2 r} \min \left(1, U^{-(k-r)}\right) \gamma(U, T)^{1-\frac{8 r}{p}} .
$$

Proof. Set $B=B(U, T)$ and $\gamma=\gamma(U, T)$. For $l \geq 0$ define

$$
B_{l}=\left\{\alpha \in B\left|2^{-l-1} \leq\right| S_{N}(\alpha) \mid \leq 2^{-l}\right\} .
$$

If $L$ denotes the least non negative integer such that $\gamma \geq 2^{-L-1}$ then $\left|S_{N}(\alpha)\right| \leq \gamma \leq 2^{-L}$ and for any $M \geq L$

$$
B=\bigcup_{l=L}^{M} B_{l} \cup D_{M},
$$

where $D_{M}=\left\{\alpha \in B|| S_{N}(\alpha) \mid \leq 2^{-M-1}\right\}$. By Lemma 2.2

$$
\left|S_{N}(\alpha) S_{N}(\alpha+\epsilon)\right| \leq C \mu(|\epsilon|)^{p}
$$

with some constant $C$ depending on $Q_{1}, \ldots, Q_{r}$. By considering $C^{-1 / 2} S_{N}(\alpha)$ instead of $S_{N}(\alpha)$ we may assume $C=1$. If $\alpha \in B_{l}$ and $\alpha+\epsilon \in B_{l}$ it follows that

$$
4^{-l-1} \leq\left|S_{N}(\alpha) S_{N}(\alpha+\epsilon)\right| \leq \mu(|\epsilon|)^{p} .
$$

If $|\epsilon| \leq N^{-1}$ this implies $|\epsilon| \leq N^{-2} 2^{4(l+1) / p}=\delta$, say, and if $|\epsilon| \geq N^{-1}$ this implies $|\epsilon| \geq 2^{-4(l+1) / p}=\rho$, say. Note that $\delta \leq \rho$ if $2^{8(l+1) / p} \leq N^{2}$, and this is true for all $l \leq M$ if

$$
M+1 \leq \log \left(N^{p / 4}\right) / \log 2 .
$$

We choose $M$ as the largest integer less or equal to $\log \left(N^{2 r} \gamma^{\frac{8 r}{p}-1}\right) / \log 2-1$. Then the assumption $\gamma \geq 4^{p /(8 r)} N^{-p / 4}$ implies $L \leq M,(4.1)$ and

$$
2^{-M} \ll N^{-2 r} \gamma^{1-8 r / p}
$$

To estimate the integral over $B_{l}$ we split $B_{l}$ in a finite number of subsets. If $B_{l} \neq \emptyset$ choose any $\beta_{1} \in B_{l}$ and set $B_{l}\left(\beta_{1}\right)=\left\{\alpha \in B_{l}|| \alpha-\beta_{1} \mid \leq \delta\right\}$. If $\alpha \in B_{l} \backslash B_{l}\left(\beta_{1}\right)$ then $\left|\alpha-\beta_{1}\right| \geq \rho$. If $B_{l} \backslash B_{l}\left(\beta_{1}\right) \neq \emptyset$ choose $\beta_{2} \in B_{l} \backslash B_{l}\left(\beta_{1}\right)$ and set $B_{l}\left(\beta_{2}\right)=\left\{\alpha \in B_{l} \backslash B_{l}\left(\beta_{1}\right)|| \alpha-\beta_{2} \mid \leq \delta\right\}$. Then $\left|\alpha-\beta_{1}\right| \geq \rho$ and $\left|\alpha-\beta_{2}\right| \geq \rho$ for all $\alpha \in B_{l} \backslash\left\{B_{l}\left(\beta_{1}\right) \cup B_{l}\left(\beta_{2}\right)\right\}$. Especially $\left|\beta_{1}-\beta_{2}\right| \geq$ $\rho$. In this way we construct a sequence $\beta_{1}, \ldots, \beta_{m}$ of points in $B_{l}$ with $\left|\beta_{i}-\beta_{j}\right| \geq \rho$ for $i \neq j$. This construction terminates after finitely many 
steps. To see this note that the balls $K_{\rho / 2}\left(\beta_{i}\right)$ with center $\beta_{i}$ and radius $\rho / 2$ are disjoint and contained in a ball with center 0 and radius $T+\rho / 2$. Thus $m \operatorname{vol}\left(K_{\rho / 2}\right) \leq \operatorname{vol}\left(K_{T+\rho / 2}\right)$ and this implies $m \ll(1+T / \rho)^{r}$. Since $B_{l} \subseteq \biguplus_{i=1}^{m} B_{l}\left(\beta_{i}\right) \subseteq \biguplus_{i=1}^{m} K_{\delta}\left(\beta_{i}\right)$ we obtain

$$
\begin{aligned}
\int_{B_{l}}\left|S_{N}(\alpha)\right| h(\alpha) d \alpha & \leq 2^{-l} \sum_{i=1}^{m} \int_{K_{\delta}\left(\beta_{i}\right)}(1+|\alpha|)^{-k} d \alpha \\
& \ll 2^{-l} \sum_{\substack{i \leq m \\
\left|\beta_{i}\right| \leq 1}} \delta^{r}+2^{-l} \sum_{\substack{i \leq m \\
\left|\beta_{i}\right|>1}}\left(\frac{\delta}{\rho}\right)^{r} \int_{K_{\rho / 2}\left(\beta_{i}\right)}|\alpha|^{-k} d \alpha .
\end{aligned}
$$

Note that $|\alpha| \asymp\left|\beta_{i}\right|$ for $\alpha \in K_{\rho}\left(\beta_{i}\right)$ if $\left|\beta_{i}\right| \geq 1$. If $U>1$ the first sum is empty and the second sum is $\ll(\delta / \rho)^{r} \int_{|\alpha|>U / 2}|\alpha|^{-k} d \alpha \ll(\delta / \rho)^{r} U^{-(k-r)}$. If $U \leq 1$ then the first sum contains $\ll \rho^{-r}$ summands; Thus both sums are bounded by $(\delta / \rho)^{r}$. This yields

$$
\int_{B_{l}}\left|S_{N}(\alpha)\right| h(\alpha) d \alpha \ll 2^{-l}\left(\frac{\delta}{\rho}\right)^{r} \min \left(1, U^{-(k-r)}\right) .
$$

Altogether we obtain by (4.2) and the definition of $\delta, \rho, L$

$$
\begin{aligned}
\int_{B}\left|S_{N}(\alpha)\right| h(\alpha) d \alpha & \ll \sum_{l=L}^{M} 2^{-l}\left(\frac{\delta}{\rho}\right)^{r} \min \left(1, U^{-(k-r)}\right)+2^{-M} \int_{|\alpha| \geq U} h(\alpha) d \alpha \\
& \ll\left(N^{-2 r} \sum_{l=L}^{M} 2^{-l(1-8 r / p)}+2^{-M}\right) \min \left(1, U^{-(k-r)}\right) \\
& \ll\left(N^{-2 r} 2^{-L(1-8 r / p)}+2^{-M}\right) \min \left(1, U^{-(k-r)}\right) \\
& \ll N^{-2 r} \gamma^{1-8 r / p} \min \left(1, U^{-(k-r)}\right) .
\end{aligned}
$$

\section{Proof of Theorem 1.1}

We apply a variant of the Davenport-Heilbronn circle method to count weighted solutions of (1.1). Without loss of generality we may assume $\epsilon=1$. Otherwise apply Theorem 1.1 to the forms $\epsilon^{-1} Q_{i}$. We choose an even probability density $\chi$ with support in $[-1,1]$ and $\chi(x) \geq 1 / 2$ for $|x| \leq 1 / 2$. By choosing $\chi$ sufficiently smooth we may assume that its Fourier transform satisfies $\widehat{\chi}(t)=\int \chi(x) e(t x) d x \ll(1+|t|)^{-r-3}$. Set

$$
K\left(v_{1}, \ldots, v_{r}\right)=\prod_{i=1}^{r} \chi\left(v_{i}\right) .
$$


Then $\widehat{K}(\alpha)=\prod_{i=1}^{r} \widehat{\chi}\left(\alpha_{i}\right)$. By Fourier inversion we obtain for an integer parameter $N \geq 1$

$$
\begin{aligned}
A(N) & :=\sum_{x \in \mathbb{Z}^{s}} w_{N}(x) K\left(Q_{1}(x), \ldots, Q_{r}(x)\right) \\
& =\sum_{x \in \mathbb{Z}^{s}} w_{N}(x) \int_{\mathbb{R}^{r}} e\left(\alpha_{1} Q_{1}(x)+\cdots+\alpha_{r} Q_{r}(x)\right) \widehat{K}(\alpha) d \alpha_{1} \ldots d \alpha_{r} \\
& =\int_{\mathbb{R}^{r}} S_{N}(\alpha) \widehat{K}(\alpha) d \alpha .
\end{aligned}
$$

Our aim is to prove for $N \geq N_{0}$, say,

$$
A(N) \geq c N^{-2 r}
$$

with some constant $c>0$. This certainly implies the existence of a nontrivial solution of (1.1), since the contribution of the trivial solution $x=0$ to $A(N)$ is $\ll N^{-s}$ and $s \geq p>8 r$. To prove (5.1) we divide $\mathbb{R}^{r}$ in a major arc, a minor arc and a trivial arc. For $\delta>0$ set

$$
\begin{aligned}
\mathfrak{M} & =\left\{\alpha \in \mathbb{R}^{r}|| \alpha \mid<N^{\delta-2}\right\}, \\
\mathfrak{m} & =\left\{\alpha \in \mathbb{R}^{r}\left|N^{\delta-2} \leq\right| \alpha \mid \leq T_{1}(N)\right\}, \\
\mathfrak{t} & =\left\{\alpha \in \mathbb{R}^{r}|| \alpha \mid>T_{1}(N)\right\},
\end{aligned}
$$

where $T_{1}(N)$ denotes the function of Lemma 3.3. Using the bound $\widehat{K}(\alpha) \ll$ $(1+|\alpha|)^{-r-3}$, Lemma 4.1 (with the choice $U=T_{1}(N)$ and the trivial estimate $\left.\gamma\left(T_{1}(N), \infty\right) \leq 1\right)$ implies

$$
\int_{\mathfrak{t}} S_{N}(\alpha) \widehat{K}(\alpha) d \alpha=O\left(N^{-2 r} T_{1}(N)^{-3}\right)=o\left(N^{-2 r}\right) .
$$

Furthermore, Lemma 4.1 with $U=N^{\delta-2}$ and $T=T_{1}(N)$, together with Lemma 3.3 yield

$$
\int_{\mathfrak{m}} S_{N}(\alpha) \widehat{K}(\alpha) d \alpha=O\left(N^{-2 r} \gamma\left(N^{\delta-2}, T_{1}(N)\right)^{1-\frac{8 r}{p}}\right)=o\left(N^{-2 r}\right) .
$$

Thus (5.1) follows if we can prove that the contribution of the major arc is

$$
\int_{\mathfrak{M}} S_{N}(\alpha) \widehat{K}(\alpha) d \alpha \gg N^{-2 r}
$$

\section{The major arc}

Lemma 6.1. Assume that each form in the real pencil of $Q_{1}, \ldots, Q_{r}$ has rank $\geq p$. Let $g, h: \mathbb{R}^{s} \rightarrow \mathbb{C}$ be measurable functions with $|g| \leq 1$ and $|h| \leq 1$. Then for $N \geq 1$

$$
N^{-2 s} \int_{[-N, N]^{s}} \int_{[-N, N]^{s}} g(x) h(y) e\left(\left\langle x, Q_{\alpha} y\right\rangle\right) d x d y \ll\left(|\alpha|^{-1 / 2} N^{-1}\right)^{p} .
$$


Proof. Note that the bound is trivial for $|\alpha| \leq N^{-2}$. Hence we assume $|\alpha| \geq N^{-2}$. Denote by $\lambda_{1}, \ldots, \lambda_{s}$ the eigenvalues of $Q_{\alpha}$ ordered in such a way that $\left|\lambda_{1}\right| \geq \cdots \geq\left|\lambda_{s}\right|$. Then $Q_{\alpha}=U^{T} \Lambda U$, where $U$ is orthogonal and $\Lambda=\operatorname{diag}\left(\lambda_{1}, \ldots, \lambda_{s}\right)$. Write $x=(\underline{x}, \bar{x})$, where $\underline{x}=\left(x_{1}, \ldots, x_{p}\right)$ and $\bar{x}=\left(x_{p+1}, \ldots, x_{s}\right)$. Then

$$
\begin{aligned}
& N^{-2 s} \int_{[-N, N]^{s}} \int_{[-N, N]^{s}} g(x) h(y) e\left(\left\langle x, Q_{\alpha} y\right\rangle\right) d x d y \\
= & N^{-2 s} \int_{U[-N, N]^{s}} \int_{U[-N, N]^{s}} g\left(U^{-1} x\right) h\left(U^{-1} y\right) e(\langle x, \Lambda y\rangle) d x d y \\
= & N^{-2(s-p)} \int_{\substack{|\bar{x}|_{\infty} \leq \sqrt{s} N \\
|\bar{y}|_{\infty} \leq \sqrt{s} N}} e\left(\sum_{i=p+1}^{s} \lambda_{i} x_{i} y_{i}\right) J(\bar{x}, \bar{y}) d \bar{x} d \bar{y},
\end{aligned}
$$

where

$$
J(\bar{x}, \bar{y})=N^{-2 p} \int_{[-\sqrt{s} N, \sqrt{s} N]^{p}} \int_{[-\sqrt{s} N, \sqrt{s} N]^{p}} \tilde{g}(\underline{x}) \tilde{h}(\underline{y}) e\left(\sum_{i=1}^{p} \lambda_{i} x_{i} y_{i}\right) d \underline{x} d \underline{y} .
$$

Here $\tilde{g}(\underline{x})=g\left(U^{-1} x\right) I_{A(\bar{x})}(\underline{x})$ with

$$
A(\bar{x})=\left\{\underline{x} \in \mathbb{R}^{p} \mid(\underline{x}, \bar{x}) \in U[-N, N]^{s}\right\} \subseteq[-\sqrt{s} N, \sqrt{s} N]^{p},
$$

and $\tilde{h}$ is defined similarly. If $|\alpha| \geq N^{-2}$ then by (2.6) $\left|\lambda_{i}\right| \asymp|\alpha| \gg N^{-2}$ for $i \leq p$. Now we apply the double large sieve bound (2.3). For $1 \leq j \leq p$ set $S_{j}=T_{j}=\sqrt{s\left|\lambda_{j}\right|} N$. Let $\mu=\nu$ be the continuous uniform probability distribution on $\prod_{j=1}^{p}\left[-T_{j}, T_{j}\right]$ and set $\bar{g}(\underline{x})=\tilde{g}\left(\left|\lambda_{1}\right|^{-1 / 2} x_{1}, \ldots,\left|\lambda_{p}\right|^{-1 / 2} x_{p}\right)$ and $\bar{h}(\underline{x})=\tilde{h}\left(\operatorname{sgn}\left(\lambda_{1}\right)\left|\lambda_{1}\right|^{-1 / 2} x_{1}, \ldots, \operatorname{sgn}\left(\lambda_{p}\right)\left|\lambda_{p}\right|^{-1 / 2} x_{p}\right)$. Then

$$
\begin{aligned}
|J(\bar{x}, \bar{y})|^{2} & \ll\left|\iint \bar{g}(\underline{x}) \bar{h}(\underline{y}) d \mu(\underline{x}) d \nu(\underline{y})\right|^{2} \\
& \ll \prod_{j=1}^{p}\left(1+\left|\lambda_{j}\right| N^{2}\right)\left(\left|\lambda_{j}\right|^{-1} N^{-2}\right)^{2} \\
& \ll|\alpha|^{-p} N^{-2 p} .
\end{aligned}
$$

Together with (6.1) this proves the lemma.

For $\alpha \in \mathfrak{M}$ we want to approximate $S_{N}(\alpha)$ by

$$
G_{0}(\alpha)=\int \sum_{x \in \mathbb{Z}^{s}} w_{N}(x) e\left(Q_{\alpha}(x+z)\right) d \pi(z),
$$

where $\pi=I_{B} * I_{B} * I_{B} * I_{B}$ is the fourfold convolution of the continuous uniform distribution on $B=(-1 / 2,1 / 2]^{s}$. Set $g(u)=e\left(Q_{\alpha}(u)\right)$. Denote by 
$g_{u_{1}}$ the directional derivative of $g$ in direction $u_{1}$, and set $g_{u_{1} u_{2}}=\left(g_{u_{1}}\right)_{u_{2}}$. We use the Taylor series expansions

$$
\begin{aligned}
& f(1)=f(0)+\int_{0}^{1} f^{\prime}(\tau) d \tau, \\
& f(1)=f(0)+f^{\prime}(0)+\int_{0}^{1}(1-\tau) f^{\prime \prime}(\tau) d \tau, \\
& f(1)=f(0)+f^{\prime}(0)+\frac{1}{2} f^{\prime \prime}(0)+\frac{1}{2} \int_{0}^{1}(1-\tau)^{2} f^{\prime \prime \prime}(\tau) d \tau .
\end{aligned}
$$

Applying the third of these relations to $f(\tau)=g\left(x+\tau u_{1}\right)$, the second to $f(\tau)=g_{u_{1}}\left(x+\tau u_{2}\right)$ and the first to $f(\tau)=g_{u_{1} u_{i}}\left(x+\tau u_{3}\right)$ we find for $u_{1}, u_{2}, u_{3} \in \mathbb{R}^{s}$

$$
\begin{aligned}
g\left(x+u_{1}\right) & =g(x)+g_{u_{1}}(x)+\frac{1}{2} g_{u_{1} u_{1}}(x)+\frac{1}{2} \int_{0}^{1}(1-\tau)^{2} g_{u_{1} u_{1} u_{1}}\left(x+\tau u_{1}\right) d \tau, \\
g_{u_{1}}\left(x+u_{2}\right) & =g_{u_{1}}(x)+g_{u_{1} u_{2}}(x)+\int_{0}^{1}(1-\tau) g_{u_{1} u_{2} u_{2}}\left(x+\tau u_{2}\right) d \tau, \\
g_{u_{1} u_{i}}\left(x+u_{3}\right) & =g_{u_{1} u_{i}}(x)+\int_{0}^{1} g_{u_{1} u_{i} u_{3}}\left(x+\tau u_{3}\right) d \tau .
\end{aligned}
$$

Together we obtain the expansion

$$
\begin{aligned}
g(x)= & g\left(x+u_{1}\right)-g_{u_{1}}\left(x+u_{2}\right)-\frac{1}{2} g_{u_{1} u_{1}}\left(x+u_{3}\right)+g_{u_{1} u_{2}}\left(x+u_{3}\right) \\
& +\int_{0}^{1}\left\{-g_{u_{1} u_{2} u_{3}}\left(x+\tau u_{3}\right)+\frac{1}{2} g_{u_{1} u_{1} u_{3}}\left(x+\tau u_{3}\right)\right. \\
& \left.+(1-\tau) g_{u_{1} u_{2} u_{2}}\left(x+\tau u_{2}\right)-\frac{1}{2}(1-\tau)^{2} g_{u_{1} u_{1} u_{1}}\left(x+\tau u_{1}\right)\right\} d \tau .
\end{aligned}
$$

Multiplying with $w_{N}(x)$, summing over $x \in \mathbb{Z}^{s}$, and integrating $u_{1}, u_{2}, u_{3}$ with respect to the probability measure $\pi$ yields

$$
S_{N}(\alpha)=G_{0}(\alpha)+G_{1}(\alpha)+G_{2}(\alpha)+G_{3}(\alpha)+R(\alpha),
$$

where $G_{0}(\alpha)$ is defined by $(6.2)$,

$$
\begin{aligned}
& G_{1}(\alpha)=-\iint \sum_{x \in \mathbb{Z}^{s}} w_{N}(x) g_{u}(x+z) d \pi(u) d \pi(z), \\
& G_{2}(\alpha)=-\frac{1}{2} \iint \sum_{x \in \mathbb{Z}^{s}} w_{N}(x) g_{u u}(x+z) d \pi(u) d \pi(z), \\
& G_{3}(\alpha)=\iiint \sum_{x \in \mathbb{Z}^{s}} w_{N}(x) g_{u v}(x+z) d \pi(u) d \pi(v) d \pi(z),
\end{aligned}
$$

and

$$
R(\alpha) \ll \sup _{|u|_{\infty},|v|_{\infty},|w|_{\infty},|z|_{\infty} \leq 1}\left|\sum_{x \in \mathbb{Z}^{s}} w_{N}(x) g_{u v w}(x+z)\right| .
$$


An elementary calculation yields

$$
\begin{aligned}
g_{u}(x)= & 4 \pi i e\left(Q_{\alpha}(x)\right)\left\langle x, Q_{\alpha} u\right\rangle, \\
g_{u v}(x)= & (4 \pi i)^{2} e\left(Q_{\alpha}(x)\right)\left\langle x, Q_{\alpha} u\right\rangle\left\langle x, Q_{\alpha} v\right\rangle+4 \pi i e\left(Q_{\alpha}(x)\right)\left\langle u, Q_{\alpha} v\right\rangle, \\
g_{u v w}(x)= & (4 \pi i)^{3} e\left(Q_{\alpha}(x)\right)\left\langle x, Q_{\alpha} u\right\rangle\left\langle x, Q_{\alpha} v\right\rangle\left\langle x, Q_{\alpha} w\right\rangle+(4 \pi i)^{2} e\left(Q_{\alpha}(x)\right) \times \\
& \left(\left\langle x, Q_{\alpha} v\right\rangle\left\langle u, Q_{\alpha} w\right\rangle+\left\langle x, Q_{\alpha} u\right\rangle\left\langle v, Q_{\alpha} w\right\rangle+\left\langle x, Q_{\alpha} w\right\rangle\left\langle u, Q_{\alpha} v\right\rangle\right) .
\end{aligned}
$$

Since $g_{u}$ and $g_{u v}$ are sums of odd functions (in at least one of the components of $u$ ) we infer $G_{1}(\alpha)=0$ and $G_{3}(\alpha)=0$. Furthermore, the trivial bound $g_{u v w}(x) \ll|\alpha|^{3} N^{3}+|\alpha|^{2} N$ for $|x|_{\infty} \ll N$ yields

$$
R(\alpha) \ll|\alpha|^{3} N^{3}+|\alpha|^{2} N .
$$

This is sharp enough to prove

$$
\begin{aligned}
\int_{\mathfrak{M}}|R(\alpha) \widehat{K}(\alpha)| d \alpha & \ll \int_{|\alpha| \leq N^{\delta-2}}|\alpha|^{3} N^{3}+|\alpha|^{2} N d \alpha \\
& \ll \int_{0}^{N^{\delta-2}} u^{r+2} N^{3}+u^{r+1} N d u \\
& \ll N^{3-(2-\delta)(r+3)}+N^{1-(2-\delta)(r+2)} \\
& \ll N^{-2 r-3+\delta(r+3)}=o\left(N^{-2 r}\right) .
\end{aligned}
$$

To deal with $G_{0}$ and $G_{2}$ we need a bound for

$$
\widetilde{G}_{j}(\alpha, u)=\int_{\mathbb{R}^{s}} \sum_{x \in \mathbb{Z}^{s}} w_{N}(x) L(x+z)^{j} e\left(Q_{\alpha}(x+z)\right) d \pi(z),
$$

where $L(x)=\left\langle x, Q_{\alpha} u\right\rangle$ and $0 \leq j \leq 2$. Using the definition of $w_{N}$ and $\pi$ we find that $\widetilde{G}_{j}(\alpha, u)$ is equal to

$$
\begin{aligned}
& \int_{B_{x_{1}}^{4}, \ldots, x_{4} \in \mathbb{Z}^{s}} \prod_{i=1}^{4} p_{N}\left(x_{i}\right) L\left(\sum_{i=1}^{4}\left(x_{i}+z_{i}\right)\right)^{j} e\left(Q_{\alpha}\left(\sum_{i=1}^{4}\left(x_{i}+z_{i}\right)\right)\right) d z_{1} \ldots d z_{4} \\
= & (2 N+1)^{-4 s} \int_{\left|x_{1}\right|_{\infty}, \ldots,\left|x_{4}\right|_{\infty} \leq N+1 / 2} L\left(\sum_{i=1}^{4} x_{i}\right)^{j} e\left(Q_{\alpha}\left(\sum_{i=1}^{4} x_{i}\right)\right) d x_{1} \ldots d x_{4} .
\end{aligned}
$$

Expanding $L\left(x_{1}+x_{2}+x_{3}+x_{4}\right)$ and $Q_{\alpha}\left(x_{1}+x_{2}+x_{3}+x_{4}\right)$ this can be bounded by

$$
\begin{array}{r}
\max _{l_{1}+l_{2}+l_{3}+l_{4}=j} N^{-4 s}\left|\int\left\{\prod_{i=1}^{4} L\left(x_{i}\right)^{l_{i}} e\left(Q_{\alpha}\left(x_{i}\right)\right)\right\} e\left(2 \sum_{i<j}\left\langle x_{i}, Q_{\alpha} x_{j}\right\rangle\right) d x_{1} \ldots d x_{4}\right| \\
\ll \max _{l_{1}+l_{2}+l_{3}+l_{4}=j} \frac{(|\alpha| N)^{j}}{N^{4 s}}\left|\int\left\{\prod_{i=1}^{4} h_{i}\left(x_{i}\right)\right\} e\left(2 \sum_{i<j}\left\langle x_{i}, Q_{\alpha} x_{j}\right\rangle\right) d x_{1} \ldots d x_{4}\right| .
\end{array}
$$


Here

$$
h_{i}\left(x_{i}\right)=L\left(x_{i}\right)^{l_{i}} e\left(Q_{\alpha}\left(x_{i}\right)\right)(|\alpha| N)^{-l_{i}} I_{\left\{\left|x_{i}\right| \leq N+1 / 2\right\}} \ll 1 .
$$

Applying Lemma 6.1 to the double integral over $x_{1}$ and $x_{2}$ and estimating the integral over $x_{3}$ and $x_{4}$ trivially we obtain uniformly in $|u| \ll 1$

$$
\widetilde{G}_{j}(\alpha, u) \ll(|\alpha| N)^{j}|\alpha|^{-p / 2} N^{-p} .
$$

Setting

$$
H_{j}(N)=\int_{\mathbb{R}^{r}} G_{j}(\alpha) \widehat{K}(\alpha) d \alpha
$$

we conclude for sufficiently small $\delta>0$ and $p>8 r\left(G_{0}(\alpha)=\widetilde{G}_{0}(\alpha, 0)\right)$

$$
\begin{aligned}
\int_{\mathfrak{M}} G_{0}(\alpha) \widehat{K}(\alpha) d \alpha & =H_{0}(N)-\int_{|\alpha| \geq N^{\delta-2}} \widetilde{G}_{0}(\alpha, 0) \widehat{K}(\alpha) d \alpha \\
& =H_{0}(N)+O\left(N^{-p}\left(\int_{N^{\delta-2} \leq|\alpha| \leq 1}|\alpha|^{-p / 2} d \alpha+1\right)\right) \\
& =H_{0}(N)+O\left(N^{-p-(2-\delta)(r-p / 2)}\right)+O\left(N^{-p}\right) \\
& =H_{0}(N)+o\left(N^{-2 r}\right) .
\end{aligned}
$$

Similarly, the explicit expression of $g_{u u}(x)$ and the definition of $\tilde{G}_{j}(\alpha, u)$ yield

$$
\begin{aligned}
& \int_{\mathfrak{M}} G_{2}(\alpha) \widehat{K}(\alpha) d \alpha \\
&= H_{2}(N)+O\left(\sup _{|u|_{\infty} \leq 2} \int_{|\alpha| \geq N^{\delta-2}}\left|\widetilde{G}_{2}(\alpha, u) \widehat{K}(\alpha)\right|+|\alpha|\left|\tilde{G}_{0}(\alpha, u) \widehat{K}(\alpha)\right| d \alpha\right) \\
&=H_{2}(N)+O\left(N^{2-p}\left(\int_{N^{\delta-2} \leq|\alpha| \leq 1}|\alpha|^{2-p / 2} d \alpha+1\right)\right) \\
& \quad+O\left(N^{-p}\left(\int_{N^{\delta-2} \leq|\alpha| \leq 1}|\alpha|^{1-p / 2} d \alpha+1\right)\right) \\
& \quad=H_{2}(N)+o\left(N^{-2 r}\right) .
\end{aligned}
$$

Hence

$$
\int_{\mathfrak{M}} S_{N}(\alpha) \widehat{K}(\alpha) d \alpha=H_{0}(N)+H_{2}(N)+o\left(N^{-2 r}\right) .
$$

Altogether we have proved that for $p>8 r$

$$
A(N)=H_{0}(N)+H_{2}(N)+o\left(N^{-2 r}\right) .
$$




\section{Analysis of the terms $H_{0}(N)$ and $H_{2}(N)$}

Lemma 7.1. Denote by $\pi_{N}$ the fourfold convolution of the continuous uniform probability distribution on $B_{N}=(-N-1 / 2, N+1 / 2]^{s}$ and by $f_{N}$ the density of $\pi_{N}$. Then

$$
H_{0}(N)=\int K\left(Q_{1}(x), \ldots, Q_{r}(x)\right) f_{N}(x) d x
$$

and

$$
H_{2}(N)=-\frac{1}{6} \int K\left(Q_{1}(x), \ldots, Q_{r}(x)\right) \Delta f_{N}(x) d x,
$$

where $\Delta f_{N}(x)=\sum_{i=1}^{s} \frac{\partial^{2} f_{N}}{\partial x_{i}^{2}}(x)$. Furthermore, $\Delta f_{N}(x) \ll N^{-s-2}$.

Proof. By Fourier inversion and the definition of $w_{N}$ and $\pi=\pi_{0}$ we find

$$
\begin{aligned}
H_{0}(N) & =\int_{\mathbb{R}^{r}} G_{0}(\alpha) \widehat{K}(\alpha) d \alpha \\
& =\int \sum_{x \in \mathbb{Z}^{s}} w_{N}(x) \int_{\mathbb{R}^{r}} e\left(Q_{\alpha}(x+z)\right) \widehat{K}(\alpha) d \alpha d \pi(z) \\
& =\int \sum_{x \in \mathbb{Z}^{s}} w_{N}(x) K\left(Q_{1}(x+z), \ldots, Q_{r}(x+z)\right) d \pi(z) \\
& =\int K\left(Q_{1}(x), \ldots, Q_{r}(x)\right) d \pi_{N}(x) .
\end{aligned}
$$

This proves the first assertion of the Lemma. Similarly,

$$
-2 G_{2}(\alpha)=\iint g_{u u}(x) d \pi(u) d \pi_{N}(x) .
$$

This implies

$$
-2 H_{2}(N)=-2 \int G_{2}(\alpha) \widehat{K}(\alpha) d \alpha=\iiint_{\mathbb{R}^{r}} g_{u u}(x) \widehat{K}(\alpha) d \alpha d \pi(u) d \pi_{N}(x) .
$$

With the abbreviations $L_{m}=2\left\langle x, Q_{m} u\right\rangle$ and $\widetilde{L}_{m}=2\left\langle u, Q_{m} v\right\rangle$ the innermost integral can be calculated as

$$
\begin{aligned}
\int_{\mathbb{R}^{r}} & g_{u u}(x) \widehat{K}(\alpha) d \alpha \\
& =\int_{\mathbb{R}^{r}} e\left(Q_{\alpha}(x)\right)\left\{\sum_{m, n=1}^{r} L_{m} L_{n} \frac{\widehat{\partial^{2} K}}{\partial v_{m} \partial v_{n}}(\alpha)+\sum_{m=1}^{r} \widetilde{L}_{m} \frac{\widehat{\partial K}}{\partial v_{m}}(\alpha)\right\} d \alpha \\
& =\sum_{m, n=1}^{r} L_{m} L_{n} \frac{\partial^{2} K}{\partial v_{m} \partial v_{n}}\left(Q_{1}(x), \ldots, Q_{r}(x)\right)+\sum_{m=1}^{r} \widetilde{L}_{m} \frac{\partial K}{\partial v_{m}}\left(Q_{1}(x), \ldots, Q_{r}(x)\right) .
\end{aligned}
$$


Here we used the relations

$$
\begin{aligned}
\frac{\widehat{\partial K}}{\partial v_{m}}(\alpha) & =2 \pi i \alpha_{m} \widehat{K}(\alpha), \\
\frac{\widehat{\partial^{2} K}}{\partial v_{m} \partial v_{n}}(\alpha) & =(2 \pi i)^{2} \alpha_{m} \alpha_{n} \widehat{K}(\alpha) .
\end{aligned}
$$

Since

$$
\begin{aligned}
& \sum_{i, j=1}^{s} u_{i} u_{j} \frac{\partial^{2}}{\partial x_{i} \partial x_{j}}\left(K\left(Q_{1}(x), \ldots, Q_{r}(x)\right)\right) \\
& =\sum_{m, n=1}^{r} L_{m} L_{n} \frac{\partial^{2} K}{\partial v_{m} \partial v_{n}}\left(Q_{1}(x), \ldots, Q_{r}(x)\right)+\sum_{m=1}^{r} \widetilde{L}_{m} \frac{\partial K}{\partial v_{m}}\left(Q_{1}(x), \ldots, Q_{r}(x)\right)
\end{aligned}
$$

we find

$$
\int_{\mathbb{R}^{r}} g_{u u}(x) \widehat{K}(\alpha) d \alpha=\sum_{i, j=1}^{s} u_{i} u_{j} \frac{\partial^{2}}{\partial x_{i} \partial x_{j}}\left(K\left(Q_{1}(x), \ldots, Q_{r}(x)\right) .\right.
$$

Altogether we conclude

$$
\begin{aligned}
-2 H_{2}(N) & =\iint \sum_{i, j=1}^{s} u_{i} u_{j} \frac{\partial^{2}}{\partial x_{i} \partial x_{j}}\left(K\left(Q_{1}(x), \ldots, Q_{r}(x)\right)\right) d \pi(u) d \pi_{N}(x) \\
& =\sum_{i=1}^{s} \iint u_{i}^{2} \frac{\partial^{2}}{\partial x_{i}^{2}}\left(K\left(Q_{1}(x), \ldots, Q_{r}(x)\right)\right) d \pi(u) d \pi_{N}(x) \\
& =\left(\int u_{1}^{2} d \pi(u)\right) \sum_{i=1}^{s} \int \frac{\partial^{2}}{\partial x_{i}^{2}}\left(K\left(Q_{1}(x), \ldots, Q_{r}(x)\right) d \pi_{N}(x) .\right.
\end{aligned}
$$

Since $\pi_{N}$ has compact support and $f_{N}$ is two times continuously differentiable, partial integration yields

$$
\int \frac{\partial^{2}}{\partial x_{i}^{2}}\left(K\left(Q_{1}(x), \ldots, Q_{r}(x)\right) f_{N}(x) d x=\int K\left(Q_{1}(x), \ldots, Q_{r}(x)\right) \frac{\partial^{2} f_{N}}{\partial x_{i}}(x) d x .\right.
$$

This completes the proof of the second assertion of the Lemma, since $\int u_{1}^{2} d \pi(u)=1 / 3$.

Finally, we prove

$$
\frac{\partial^{2} f_{N}}{\partial x_{i}^{2}}(x) \ll N^{-s-2}
$$

Note that

$$
\widehat{f_{N}}(t)=\prod_{i=1}^{s}\left(\frac{\sin \left(\pi t_{i}(2 N+1)\right)}{\pi t_{i}(2 N+1)}\right)^{4}=\widehat{f}_{0}((2 N+1) t) .
$$


Hence, by Fourier inversion

$$
\begin{aligned}
\frac{\partial^{2} f_{N}}{\partial x_{i}^{2}}(x) & =(-2 \pi i)^{2} \int \widehat{f_{N}}(t) t_{i}^{2} e(-\langle t, x\rangle) d t \\
& =-(2 \pi)^{2}(2 N+1)^{-s-2} \int \widehat{f}_{0}(t) t_{i}^{2} e(-(2 N+1)\langle t, x\rangle) d t \\
& \ll N^{-s-2}
\end{aligned}
$$

This completes the proof of Lemma 7.1. We remark that we used the fourfold convolution in the definition of $w_{N}, \pi_{N}, f_{N}$ for the above treatment of $H_{2}(N)$ only. At all other places of the argument a twofold convolution would be sufficient for our purpose.

Lemma 7.2. Assume that the system $Q_{1}(x)=0, \ldots, Q_{r}(x)=0$ has a nonsingular real solution, then

$$
\lambda\left(\left\{\left.x \in \mathbb{R}^{s}|| Q_{i}(x)\left|\leq N^{-2},\right| x\right|_{\infty} \leq 1\right\}\right) \gg N^{-2 r},
$$

where $\lambda$ denotes the s-dimensional Lebesgue measure.

Proof. This is proved in Lemma 2 of [10]. Note that if a system of homogeneous equations $Q_{1}(x)=0, \ldots, Q_{r}(x)=0$ has a nonsingular real solution, then it has a nonsingular real solution with $|x|_{\infty} \leq 1 / 2$.

Now we complete the proof of Theorem 1.1 as follows. For $c>0$ and $N>0$ set

$$
A(c, N)=\lambda\left(\left\{\left.x \in \mathbb{R}^{s}|| Q_{i}(x)\left|\leq N^{-2},\right| x\right|_{\infty} \leq c\right\}\right) .
$$

Then

$$
A(c, N)=c^{s} A(1, c N) .
$$

By Lemma 7.1

$$
\begin{aligned}
H_{0}(N) & \gg N^{-s} \int_{|x|_{\infty} \leq 2 N} K\left(Q_{1}(x), \ldots, Q_{r}(x)\right) d x \\
& \gg \int_{|y|_{\infty} \leq 2} K\left(N^{2} Q_{1}(y), \ldots, N^{2} Q_{r}(y)\right) d y \\
& \gg A(2,2 N) \\
& \gg A(1,5 N)
\end{aligned}
$$


and

$$
\begin{aligned}
H_{2}(N) & \ll N^{-s-2} \int_{|x|_{\infty} \leq 5 N} K\left(Q_{1}(x), \ldots, Q_{r}(x)\right) d x \\
& \ll N^{-2} \int_{|y|_{\infty} \leq 5} K\left(N^{2} Q_{1}(y), \ldots, N^{2} Q_{r}(y)\right) d y \\
& \ll N^{-2} A(5, N) \\
& \ll N^{-2} A(1,5 N) .
\end{aligned}
$$

With Lemma 7.2 this yields

$$
H_{0}(N)+H_{2}(N) \gg A(1,5 N) \gg N^{-2 r}
$$

for $N \geq N_{0}$, say. Together with (6.3) this completes the proof of Theorem 1.1 .

\section{References}

[1] V. Bentkus, F. Götze, On the lattice point problem for ellipsoids. Acta Arith. 80 (1997), $101-125$.

[2] V. Bentkus, F. Götze, Lattice point problems and distribution of values of quadratic forms. Annales of Mathematics 150 (1999), 977-1027.

[3] E. Bombieri, H. Iwaniec, On the order of $\zeta(1 / 2+i t)$. Ann. Scuola Norm Sup. Pisa (4) 13 (1996), 449-472

[4] J. BRÜDERn, R.J. Cook, On simultaneous diagonal equations and inequalities. Acta Arith. 62 (1992), 125-149.

[5] H. DAvenPort, Indefinite quadratic forms in many variables (II). Proc. London Math. Soc. 8 (1958), 109-126.

[6] R. J. Cook, Simultaneous quadratic equations. Journal London Math. Soc. (2) 4 (1971), $319-326$

[7] R. Dietmann, Systems of rational quadratic forms. Arch. Math. (Basel) 82 (2004), no. 6, $507-516$.

[8] E. D. Freeman, Quadratic Diophantine Inequalities. Journal of Number Theory 89 (2001), 269-307.

[9] G. A. Margulis, Discrete subgroups and ergodic theory. In "Number Theory, Trace Fromulas and Discrete Groups (Oslo, 1987)", 377-398, Academic Press, Boston, 1989.

[10] W. M. Sснмidt, Simultaneous rational zeros of quadratic forms. Séminaire Delange-PisotPoitou (Théorie des Nombres), Paris 1980-1981, Progr. Math. 22 (1982), 281-307.

[11] H. P. F. Swinnerton-Dyer, Rational zeros of two quadratic forms. Acta Arith. 9 (1964), $261-270$.

Wolfgang MÜLLER

Institut für Statistik

Technische Universität Graz

8010 Graz, Austria

E-mail : w.mueller@tugraz.at 